\title{
Morphometric Indices of Multilocular Adipose Tissue Cells When Adapting to Cold
}

\author{
Elena D. Kolodeznikova ${ }^{1}$, Elena V. Pshennikova ${ }^{1, *}$, Irina V. Nikolaeva ${ }^{1}$ and Tuyaara \\ P. Sivtseva ${ }^{1}$
}

\begin{abstract}
${ }^{1}$ M.K. Ammosov North-Eastern Federal University, Medical Institute, 27, Oyunsky St., 677000, Yakutsk, Russia *Corresponding author.Email:el_viss@mail.ru
\end{abstract}

\begin{abstract}
The article presents the results of experimental studies of interscapular and peri-adrenal brown adipose tissue of rats kept at low temperatures. Experimental animals were kept at a temperature of $+50{ }^{\circ} \mathrm{C}$ for $1.3,7,15,30,90$ days. The skin temperature of the lateral surfaces of the body, ears, paws, tail, interscapular and rectal regions was measured to assess the overall body reactions. The studies made it possible to clarify and detect some morphological features of brown adipocytes in the processes of adaptation to cold. Changes in cell structure occurred in three periods: acute ( 1 day), transient (3-15 days) and period of steady adaptation (30-90 days). Morphometric studies of brown adipocytes of interscapular and near-adrenal brown adipose tissue showed that with an increase in the duration of stay under conditions of low temperatures, the number of destructive types of brown adipocytes decreases, cells activity increase of types II and III is observed. Under low temperature conditions, brown adipose tissue cells are rapidly incorporated into the thermoregulation and heat production process. The findings show adaptive mechanism effect for brown adipose tissue cells.
\end{abstract}

Keywords: morphometric indices, multilocular adipose tissue cells, adaptive mechanism

\section{INTRODUCTION}

In the last two decades, adipose tissue interest has increased significantly. This is due to the fact that the sources of development of these tissues were clarified. Previously, it was believed that they are formed from mesenchyma. However, recently it has been established that only white adipose tissue forms from mesenchyma. As for brown adipose tissue, it develops not from mesenchyma in embryogenesis, but from mesoderm, myotome of somites [1]. Increasing attention has been paid not only to the functions and structure of brown adipose tissue, but also to cold exposure and adaptation processes.

The process of adaptation to cold is very complex, it includes nerve-reflex and endocrine mechanisms. In this process, a specific hormone is released that enhances heat production by combustion of free fatty acids and separation of oxidative phosphorylation. Since the end of the last century, attention of many specialists in the field of morphology, biochemistry, physiology, clinics were attracted to the structure, topography and metabolism of brown adipose tissue (BAT) [2-9].

It has been proven that for animals hibernating, BAT is absolutely necessary. Theoretical calculations showed that $1.9 \mathrm{kcal} / \mathrm{kg}$ is required to warm hamsters at a temperature of 40 to $370 \mathrm{C}$, excluding heat loss to the environment. Maintenance of the necessary level of liver energy exchange is carried out with the participation of brown adipose tissue, which can produce up to $1 / 3$ of thermal energy.

The brown adipose tissue is widespread up to 10 years in a person, then its number decreases, but remains until the age of 80 .

Currently, the role of brown adipose tissue in animals and humans in the energy balance is given great importance. The thermogenic response is significantly higher after ingestion of high carbohydrate than fat 
feeding. Data showed that many rats on a high-calorie diet maintain normal body weight due to increased dietary thermogenesis, increasing energy consumption to $70 \%$ above normal. BAT is well vascularized and closely interacts with many large vessels, surrounds the entire thorax and all organs in it, as well as organs in the abdominal cavity. Large clusters of it are located in the interscapular region, between muscles in the neck region, in the axillary regions and near the adrenal glands, slightly smaller around the kidneys, in the mesentery of the small intestines and in other regions. Blood from large veins of interscapular brown adipose tissue flows into the veins of the cerebrospinal canal and warms the central nervous system.

Currently, researches quite well described ideas about the biology, cellular and molecular foundations of the functioning of BAT, a unique organ of animal organism. The uniqueness of BAT lies in the fact that heat generation in cells is carried out by a specific mechanism using the disconnecting protein $1-\mathrm{RB} 1$, is a form of physiological work and is provided by neuroendocrine regulation, like any function [9-11]. The issues related to BAT relation with other organs and systems in normal and pathology are also well studied [12-16]. But the processes of BAT change in stress states and adaptation in the conditions of the North are not sufficiently studied. When adapting to various extreme factors in the body, significant morphological and biochemical changes occur that allow survive in the new conditions of existence. However, with a long period of adaptation, BAT features require further study, which will allow a deeper understanding of the mechanism of protective and adaptive reactions of the body and the role of BAT, to develop practical recommendations for improving the population of the North, and reducing the period of adaptation of the alien population.

The aim of this article was to study alterations of adipocytes of interscapular and peri-adrenal brown adipose tissue (BAT) of rats under low temperature conditions.

\section{MATERIALS AND METHODS}

Paranadrenal and interscapular brown adipose tissue were studied in 40 laboratory animals (white rats). Experimental morphological studies were carried out with prolonged $\left(+5{ }^{\circ} \mathrm{C}\right)$ cooling $(1,3,7,15,30,90$ days). To evaluate the general reactions of the body, the skin temperature of the lateral surfaces of the body, ears, paws, tail, interscapular surface and rectal area was measured, the mass of interscapular and peritoneal brown adipose tissue was determined. The material was fixed with $10 \%$ formalin and Carnoy's fluid. Morphometric studies were carried out on histological preparations stained with azure II and hematoxylineosin, with an increase of $10 \times 40$ in each experimental group of animals. Quantitative analysis counted the number of different types of brown adipocytes in the standard field of view. A count of 1000 cells in each preparation was made, taking into account 7 types of BAT cells, their destructive forms, white adipose tissue cells and lymphocytes. For each case, the percentage of BAT cell types was detected.

Macroscopical brown adipose tissue has the form of lobed well-delimited brownish-colored formations of irregular shape. The interstitial BAT of irregular shape in the form of pyramid, facing its apex towards the spine, has a lobed structure. The outside is covered with a thin connective tissue capsule. The cytological characterization of brown adipocytes is characterized by a large variety of cell sizes and tinctorial features, which is associated primarily with the size and number of lipid inclusions, as well as the number of mitochondria. Our studies of BAT in rodent adults at the light-optical and electron-microscopic level made it possible to establish, in addition to the main types, a number of transition cell forms and classify them as follows [3].

According to Yu.I. Afanasyev and E.D. Kolodeznikova's classification, [3], several types of adipocytes (I-VII) are distinguished morphometrically in brown adipose tissue:

Type I. Malodifferentiated granular brown adipocyte, more often found in newborns, cells of irregular shape (in rodents $-10 \times 11 \mu \mathrm{m}$ ), the nucleus is large (up to $6 \mu \mathrm{m}$ ) with a uniform distribution of chromatin blocks and a large nucleus, the cytoplasm is granular or with single lipid inclusions ("dark" cells).

Type II. Young grainy-multilocular brown adipocyte, more common in young individuals. The cells are somewhat elongated (in rodents $-12 \times 15 \mu \mathrm{m}$ ), the nucleus is large, rounded, less often irregular in shape with 1-2 nuclei and a uniform distribution of chromatin blocks is located in the center. The cytoplasm is filled with lipid inclusions of the same size, between which clusters of mitochondria ("dark" cells) are visible.

Type III. Mature multilocular-grainy brown adipocyte, more common in adults. The cells are larger (in rodents $15 \times 19 \mu \mathrm{m}$ ), the nucleus with a large nucleus is somewhat offset from the center, rounded or irregular in shape, with a large concentration of chromatin blocks on the periphery. The cytoplasm is coarse-grained with lipid inclusions of various sizes (larger ones appear) (transition form from "dark" cells to "light" ones).

Type IV. Mature multivocular brown adipocyte, more common in adult animals. The cells have a different configuration (in rodents $-17 \times 23 \mu \mathrm{m}$ ), the nucleus is somewhat elongated, shifted to the periphery, with an enlightened karyoplasm. The cytoplasm is dominated by large lipid inclusions, although smaller ones remain (transition form from "dark" cells to "light" ones). 
$\mathrm{V}$ type. Locular brown adipocyte, cells of somewhat elongated shape $(18-20 \times 25-29 \mu \mathrm{m})$, the nucleus is shifted to the periphery and has oval, sometimes deformed outlines, the nucleus is not clearly detected, the cytoplasm is filled with large, closely adjacent lipid inclusions, the grain size is insignificant. These cells are kind of an energy material depot.

Type VI. Monolocular brown adipocyte, large-sized cells, the nucleus is flat and shifted to the periphery, sometimes picnotized, the cytoplasm is occupied by one or two large lipid inclusions, the cytoplasmic rim is wider than that of white adipose tissue ("light" cell).

Type VII. Cells are different and vary dramatically in configuration and diameter, there is picnosis, caryorexis, caryolysis (dystrophic changes) in the nuclei of these cells, cytoplasm is pale or intense, homogeneous, does not have grain, has few lipid inclusions.

Table 1. Temperature in rats at different cooling times

\section{RESULTS OF THE RESEARCH}

The studies made it possible to clarify and detect some ultramicroscopic data. Prolonged cooling of animals causes structural-functional changes at the organ, tissue, cellular and subcellular levels in FAT, which can be divided into three periods: acute cooling period ( 1 day), transition period (3-15 days), and a period of steady adaptation (30-90 days).

Throughout the experiment, the temperature of the skin naturally changes. After a day, it drops sharply at all measurement points. The temperature with small fluctuations remains lowered until the end of the experiment in the skin of the paws and tail. Skin temperature of the side surfaces of the trunk rises by 7-30 days, and then decreases again. Rectal temperature and brown gland temperature, starting from 3 days, becomes above the initial level and lasts up to 30 days. By 90 days it had reached the average level characteristic of intact animals (Table 1).

\begin{tabular}{|l|l|l|l|l|l|l|}
\hline $\begin{array}{l}\text { Observation } \\
\text { time, days }\end{array}$ & interscapular region & $\begin{array}{l}\text { Lateral surfaces } \\
\text { of the body }\end{array}$ & $\begin{array}{l}\text { Rectal } \\
\text { temperature }\end{array}$ & Ears & Paws & Tail \\
\hline Control & $35.6 \pm 0.6$ & $34.5 \pm 0.2$ & $35.2 \pm 0.2$ & $29.1 \pm 0.7$ & $24.6 \pm 0.4$ & $23.4 \pm 0.5$ \\
\hline 1 day & $34.2 \pm 0.5$ & $32.9 \pm 0.5$ & $33.2 \pm 0.7$ & $23.1 \pm 0.7$ & $20.5 \pm 0.5$ & $18.5 \pm 0.5$ \\
\hline 3 days & $35.7 \pm 0.3$ & $32.6 \pm 0.6$ & $35.4 \pm 0.2$ & $24.7 \pm 0.9$ & $15.8 \pm 0.7$ & $18.0 \pm 0.5$ \\
\hline 15 days & $36.4 \pm 0.2$ & $34.2 \pm 0.2$ & $36.0 \pm 0.2$ & $23.6 \pm 0.5$ & $16.0 \pm 0.6$ & $18.2 \pm 0.3$ \\
\hline 30 days & $36.4 \pm 0.2$ & $35.2 \pm 0.1$ & $37.2 \pm 0.2$ & $25.9 \pm 0.7$ & $16.8 \pm 0.6$ & $16.5 \pm 0.7$ \\
\hline 90 days & $35.5 \pm 0.5$ & $34.9 \pm 0.5$ & $35.7 \pm 0.6$ & $23.6 \pm 0.4$ & $20.8 \pm 0.5$ & $18.4 \pm 0.6$ \\
\hline
\end{tabular}

The experimental studies of interscapular region and peri-adrenal brown adipose tissue of rats, showed there is an expansion of blood capillaries and their filling with shaped blood elements after the 1st day. There is a degradation of mast cells and increase in the number of eosinophils and lymphocytes. Capillary walls become swollen and small hemorrhages occur as a result of increasing permeability. The endothelial cells of the capillaries swell and vacuolize, the cytoplasm is enlightened and filled with numerous pinocytous vesicles. Due to swelling, the BAT mass increases slightly. Dystrophic changes of brown adipocytes occur. The cytoplasm of most cells stains more intensively and becomes more compact, their size decreases to $14 \mu \mathrm{m}$. Cells contain 1-2 nuclei, they are located eccentrically, irregularly shaped, the number of nuclei increases to three, an increase in the concentration of ribonucleoproteins (RNP) is determined. Changes occur due to swelling and enlightening of the cytoplasm, the size of such cells increases to $27 \mu \mathrm{m}$ insome cells. Changes in cells are often associated with a decrease in the number and size of lipid inclusions. During this period, brown type IV adipocytes (23.6\%) and degenerative forms (25.8-27.1\%) are more defined (Fig. 1.2). Our morphological studies were supported by histochemical and ultrastructural studies [3].

When the animals are cooled for 3 days, the weight of brown adipose tissue decreases due to a decrease in lipid inclusions. Examination of histological preparations shows uneven reactive changes in the lobes of brown adipose tissue. In some slices, narrowed ones are more common, in others, dilated hemocapillars, with local hemorrhages and necrosis. Endothelial cells of capillaries and blood vessels are hypertrophied in places with a pale cytoplasm. There is an increase in mast cell degranulation and a sharp decrease in eosinophils. The lobes of FAT with dilated blood vessels are dominated by dark small cells. In other lobes where hemorrhages are noted, light, devastated cells with nuclei along the periphery are found. Glycogen disappears from adipocytes. There is an increase in the number of II-IV cell types in the BAT lobes, the proportion of destructive cells becomes less, but is still quite high (20.8-24.0 \%) (Fig.1.2). 
Table 2. Quantitative (\%) values of brown adipocyte types in rat interscapular region and peri-adrenal FAT

\begin{tabular}{|c|c|c|c|c|c|c|c|c|c|c|c|c|c|c|}
\hline \multirow{3}{*}{$\begin{array}{l}\text { Observation } \\
\text { time, days }\end{array}$} & \multicolumn{12}{|c|}{ Types of brown adipocytes } & & \\
\hline & \multicolumn{2}{|l|}{1} & \multicolumn{2}{|l|}{$\|$} & \multicolumn{2}{|l|}{ III } & \multicolumn{2}{|l|}{ IV } & \multicolumn{2}{|l|}{ V } & \multicolumn{2}{|l|}{ VI } & \multicolumn{2}{|l|}{ VII } \\
\hline & M & 0 & M & 0 & M & 0 & M & 0 & M & 0 & M & 0 & M & 0 \\
\hline Control & 12 & 20.5 & 38.6 & 28.2 & 39.1 & 35.9 & 10.3 & 15.4 & 0 & 0 & 0 & 0 & 0 & 0 \\
\hline 1 day & 6.7 & 6.8 & 9.1 & 6.8 & 13.5 & 13.6 & 23.6 & 23.7 & 14.6 & 16.9 & 6.7 & 5.1 & 25.8 & 27.1 \\
\hline 3 days & 6.2 & 4.0 & 16.7 & 13.3 & 19.8 & 19.0 & 16.7 & 20.0 & 17.7 & 17.0 & 2.1 & 2.7 & 20.8 & 24.0 \\
\hline 15 days & 3.2 & 2.9 & 20.4 & 20.9 & 17.2 & 16.6 & 27.9 & 22.4 & 19.4 & 17.9 & 2.2 & 2.9 & 9.7 & 16.4 \\
\hline 30 days & 5.5 & 4.4 & 6.6 & 25.6 & 11.0 & 8.9 & 45 & 32.2 & 9.9 & 10.0 & 7.7 & 4.5 & 14.3 & 14.4 \\
\hline 90 days & 12.4 & 13.7 & 22.5 & 20.5 & 33.7 & 23.3 & 14.6 & 19.2 & 9.0 & 12.3 & 2.2 & 5.5 & 5.6 & 5.5 \\
\hline 1 day & 4.3 & 12.2 & 61.3 & 41.5 & 23.7 & 34.1 & 5.4 & 12.2 & 2.0 & 0 & 2.2 & 0 & 1.1 & 0 \\
\hline
\end{tabular}

At the 7-15th day from the start of cooling, an increase in BAT weight is observed, which is associated with an increase in lipid inclusions that are rounded or oval in shape. Reduced number of destructive and degenerative cell forms. A cluster of young type I adipocytes (4.4-5.5\%) appears. By 15 days, BAT mass increases significantly, which is associated with an increase in cell size by 1.5-2 times (up to 35-38 microns). Lipid inclusions fill the entire cytoplasm. Many brown adipocytes in their morphology become similar to the cells of the control group of animals (intact). By number and size, brown type IV adipocytes predominate $(32.2-45.0 \%)$. Although dystrophic cells with type VII pynotic nuclei $(14.4 \%)$ are still often found (Fig. 1, 2).

The adaptation period begins on the 30-90th day. On the 30th day, BAT mass increases, the cells become hypertrophic. Dark and light adipocytes are clearly distinguished. The number of light cells prevails over dark cells. A large number of small lipid inclusions are preserved in the cells, the nuclei of the cells are somewhat elongated with large nuclei. There are few lipid inclusions in dark cells, the nucleus is oval in shape, often eccentrically located, and chromatin is uniformly located in the nucleus. During this period, an increase in the number of cells of types II (up to 41.5-61.3\%) and III (up to 23.3-34.1\%) is observed. Around BAT lobes between muscle fibers and around the kidneys, accumulations of white adipose tissue appear. Vessels of normal structure. Partial degranulation is noted in single mast cells, eosinophils are rare.

Large cells that differ from control cells, size and greater chromophilicity are determined in FAT by the end of the experiment, on the 90th day. Dark adipocytes contain, almost the same size, lipid inclusions. In light adipocytes, lipid inclusions have different sizes and shapes, among them there are larger cells with one lipid inclusion, similar to white adipose tissue cells. Morphologically, the dark adipocytes of BAT during this period are very similar to the dark cells of BAT of control animals. Lipid inclusions are variable in number and magnitude. Type II cells predominated on day 90 and there was a significant decrease in degenerative and destructive cell types (Table).

Blood vessels are dilated and contain shaped blood elements. Among the BAT cells there are many lymphocytes. The above picture of hypertrophy of BAT after three months of cold exposure in rats indicates adaptive processes.

\section{CONCLUSIONS}

The study of BAT cell changes reflects an acute cooling period (1-2 days), a transition period (3-15 days) and a period of steady adaptation (up to 90 days).

The first period of tension (1 day) is characterized by hemostasis, point hemorrhages, dystrophic change of brown adipocytes, decrease in the number and size of lipid inclusions, increase in BAT weight. The IV cell type and degenerative forms are more defined. Obviously, increased exchange of the first voltage hours leads to a decrease in energy and plastic material.

In the second (transitional) period (3-15 days), it is characterized by a mosaic of morphological processes. The BAT mass decreases, the blood filling of capillaries is uneven, foci of hemorrhages and necrosis appear. The degranulation of mast cells increases, the number of eosinophils decreases. Destructive processes are expressed on the third day and reach a maximum by the 15 th. At the same time, reparative processes in the form of clusters of young forms of type I cells and lymphocytes were noted. A shift to the left was noted an increase in adipocytes of types II-IV.

The third period (relative adaptation) is characterized by cell hypertrophy, an increase in lipid inclusions, a decrease in destructive changes, BAT mass increase and temperature increase of the interscapular region. 
Thus, morphometric studies of brown adipocytes of interscapular and peri-adrenal brown adipose tissue showed that with an increase in the duration of stay under low temperature conditions, the number of destructive types of brown adipocytes decreases. There is also an increase in the activity of type II and III cells as the time of exposure to cold increases. During all the observation periods, lymphocytes (about $6 \%$ ) are found among BAT cells. In the first days of the week, that is, during the period of inflammatory processes, lymphocytes were larger. The findings show the effect of an adaptive mechanism for adapting brown adipose tissue cells. When adapting to various extreme conditions in the body, significant biochemical and morphological changes occur that allow you to survive in the new conditions of existence. However, a long period of adaptation requires further study using modern research methods.

\section{REFERENCES}

[1] O.D. Myadelets, V.O. Myadelets, I.S. Sobolevskaya, T.N. Kichigina, Bull. of VSMU 13(5) (2014) 32-44.

[2] T.V. Murashkin, Experim. and appl. Morphol. (1988) 80-84.

[3] Yu.I. Afanasyev, E.D. Kolodeznikova, Brown adipose tissue, Publ. House of Irkutsk Univ., 1995, $184 \mathrm{p}$.

[4] O.D. Myadelets, E.P. Klushenkov, Basic and appl. Res. (2001) 334-337.

[5] L.N. Medvedev, E.I. Yelsukova, Brown adipose tissue: molecular foundations of regulated thermogenesis, Amalgama, Krasnoyarsk, 2002, $528 \mathrm{p}$.

[6] O.D. Myadelets, V.P. Adaskevich, Morphofunctional dermatology, Med. lit., Moscow, 2006, 752 p.

[7] P. Boström et al., Nature 481 (2012) 463-688.

[8] Y. Zhang et al., Diabetes 63(2) (2014) 514-525.

[9] N.I. Volkova, M.I. Porksheyan, A.N. Gulmagomedova, Med. Bull. of the South of Rus. 3 (2016) 4-13.

[10] L.N. Medvedev, E.I. Yelsukova, Succes. of physiol. Sci. 33(2) (2002) 17-29.

[11] Van Marken Lichtenbelt, Clin. Nutr. Metab. Care 15(6) (2012) 547-452.

[12] M.J. Obregon, Front. Physiol. 5(87) (2014) 479.

[13] J. Wu et. al., Genes Dev. 1.27(3) (2013) 234-250.
[14] C.D. Wrann et al., Cell. Metab. 18(5) (2013) 649659.

[15] A.J. Whittle, A. Vidal-Puig, J. Clin. Invest. 122(3) (2012) 804-807.

[16] M. Bordicchia, J. Clin. Invest. 122(3) (2012) 10221036. 